\title{
Research into structural design and simulation of the inspection robot for ultra-high voltage power transmission lines
}

\author{
Yiheng Bian ${ }^{1, a}$ \\ ${ }^{1}$ Department of electric power engineering, North China Electric Power University , Baoding of Hebei \\ province 071000, China \\ a1158082860@qq.com
}

\begin{abstract}
Keywords: inspection robot; extra-high voltage power transmission line; structural design; simulation analysis

Abstract. With the rapid development of the power system, there has been a higher requirement of the power transmission lines. However, the traditional power transmission line test usually adopts the helicopter aerial survey and artificial visual inspection, which might incur a high inspection cost but can hardly ensure the inspection precision. Under the condition, the inspection robot is developed to respond to problems with the traditional power transmission line inspection methods, having achieved significantly positive effects. This paper is an exploration and analysis of the structural design thinking of the inspection robot, the robot kinematic issues and the robot movement simulation.
\end{abstract}

\section{Introduction}

As an important technical approach for the current power transmission line test, the inspection robot can be hung on the overhead ground wire to automatically conduct power transmission line inspection. Some commonly-seen problems like guide line damage, insulator degradation and line faults can be efficiently tested. However, how to give full play to the inspection robot and do a good work of structural design to meet the power transmission line inspection requirements remains an issue of great concern. This research targets at the design requirements of the inspection robot, so it is of vital significance.

\section{Research thinking of the structural design}

The structure of the inspection robot shows obvious complex characteristics. Therefore, it is necessary to do a good work of 3D model building during the structural design. As the building of various robot parts, the CATIA software is introduced. The functions of the software include: 1) Assembly design: Introduce all assembly parts into it and meet the design, analysis and manufacturing requirements of assembly parts; 2) Engineering drawing: Directly build the engineering drawing; 3) Characteristic design module: Provide exclusive dialogues for users and guarantee prism parts design of users. Besides, the software can also introduce the curve design, the metal plate design and the assembly simulation into it.

In the practical robot structural design, the $500 \mathrm{kV}$ power transmission lines are adopted as research objects. It is required that the robot design relies on the computer control system and can move on the overhead line and automatically finish the operation of evading obstacles. To the end, the inspection robot should have an edge in terms of obstacle crossing capability and climbing capability, and its weight should preferably be controlled within $100 \mathrm{~kg}$. During the practical design process, the overall framework of the inspection robot should be clarified, which mainly includes the flexible arms, the palm opening and closing device, the braking device, the driving device, the control cabinet and the power box. The schematic diagram of the specific structure is shown in Fig. 1 below: 


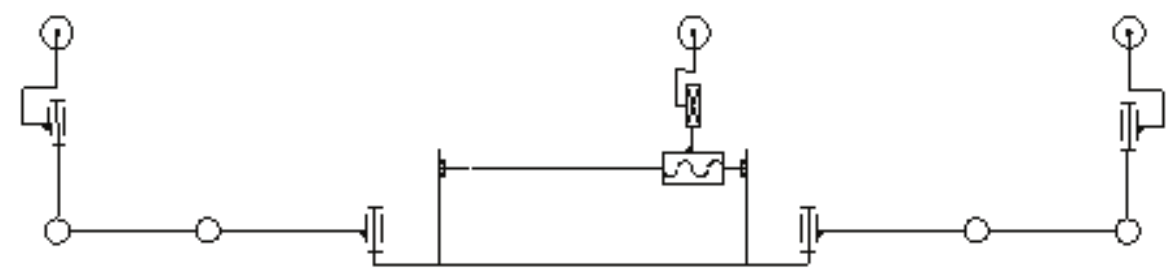

Fig. 1 Schematic diagram of the inspection robot structure

In the structure, the actuating components feature the flexible arms, which can support the end effector and the wrist of the robot to some extent. In this design, the joint-type structure dominates. Relevant movement parameters under the structure are reflected as the end effector length, the wrist length, the forearm length and the big arm length, which is $344 \mathrm{~mm}, 72 \mathrm{~mm}, 400 \mathrm{~mm}$ and $250 \mathrm{~mm}$, respectively. Besides, all joints rotate within $\pm 90^{\circ}$. Two mechanisms, the stepping-type and the wheel-type, can be introduced. The former has higher transmission efficiency; while the latter can increase the inspection speed of the robot. As to the braking device, it includes the spring guide axis, the bottom base, the upper base, the pin roll, and the fixed and flexible brake ratchets, which can prevent the robot in fault from falling down or being out of control. After functions of various parts are confirmed, the CATIA environment can be introduced to confirm the mass inertia parameters of various parts [1]. The parameters are shown in Table 1 in details.

Table 1 Mass inertia parameters of major robot parts under the CATIA model

\begin{tabular}{ccccc}
\hline Parts & Mass & Izz & Iyy & Ixx \\
\hline Flexible brake ratchet & 0.0115 & $2.094 \mathrm{e}-005$ & $1.863 \mathrm{e}-005$ & $2.375 \mathrm{e}-006$ \\
Fixed brake ratchet & 0.085 & $4.021 \mathrm{e}-005$ & $3.171 \mathrm{e}-004$ & $2.849 \mathrm{e}-004$ \\
Forearm & 0.279 & 0.005 & 0.005 & $97 \mathrm{e}-005$ \\
Rolling wheel & 1.439 & 0.002 & 0.002 & 0.003 \\
Big arm & 1.437 & 0.013 & 0.012 & 0.002 \\
\hline
\end{tabular}

\section{Analysis of kinematic issues of mechanical arms}

Analysis of the kinematic characteristics of mechanical arms is an important guarantee for reasonable design of various robot joints. During the practical analysis process, relevant mathematical theories are introduced. First, homogeneous transformation and rigid body pose analysis. The analysis method is mainly for the research into the relationship between mechanical arms and the environment or the internal components of mechanical arms. In terms of the rigid body pose, Point $\mathrm{P}$ is chosen; $\mathrm{Px}, \mathrm{Py}$ and $\mathrm{Pz}$ respectively stand for the coordinate of Point $\mathrm{P}$ in the coordinate system. Based on that, Point $\mathrm{P}$ is expressed in the vector form, and $\mathrm{w}$ is adopted as the proportional factor. Under the condition that $\mathrm{Px}=\mathrm{X} \cdot \mathrm{w}, \mathrm{Py}=\mathrm{y} \cdot \mathrm{w}$ and $\mathrm{Pz}=\mathrm{Z} \cdot \mathrm{w}$, there is $\bar{P}\left[\begin{array}{c}x \\ y \\ \mathrm{z} \\ w\end{array}\right]$. In this way, when the proportional factor, $w$, changes, pose of the referential point can be analyzed. Besides, D-H expression method can also introduced to express the kinematical equation and to make the robot connecting rod and joint design more reasonable [2].

Kinematic analysis of mechanical arms can proceed from forward kinematics, inverse kinematics, speed and Jacobian matrix. In terms of forward kinematics, the D-H expression method can be adopted for the coordinate system building and the parameter table filling. After these parameters are clarified, they can be put into relevant equations. Assume that Ai stands for the transformation matrix of adjacent joints, and that the transformation between two coordinate systems, including the end face and the substrate of robot's hands, can be expressed as RTH. Then, RTH=A1A2A3A4. After that, the pose of the hand end face in the coordinate system can be deduced. In terms of the inverse kinematics, the angle solution is finished through decoupling in different angles. For example, $\mathrm{A} 1-1 \mathrm{RTH}=\mathrm{A} 2 \mathrm{~A} 3 \mathrm{~A} 4$ can be directly employed to deduce the joint rotational angle. Besides, in terms 
of the component changes, the Jacobian matrix can be used for expression. Generally speaking, the value of the joint corner can be expressed as $[\mathrm{D}]=[\mathrm{J}][\mathrm{D} \theta]$ when time changes. The Jacobian matrix is expressed as $\mathrm{J}$, and the joint differential movements can be expressed as D $\theta$. The differential movement of the coordinate system is D. Through the equation the joint angular velocity can be solved [3].

\section{Robot movement simulation from the perspective of ADAMS}

During the practical simulation process, the mechanism movement situations are confirmed through the simulation software. During the simulation process, this paper introduces ADAMS many-body dynamics into it, which can integrate ODE solution, nonlinear solution and linear solution into it. The simulation can be subdivided into: 1) Model building: In this step, the part model should be built, and the model should be set with corresponding acting force and restraint; 2) Model test: The measurement should be defined quantitatively. Based on that, the model is simulated, and it should be judged whether the acting force, restraint and parts meet the simulation requirements; 3 ) Model validation: Simulation results and test results are compared and the model is judged to see whether it is reasonable; 4) Model refinement: The complex elements are introduced into the model, such as flexible connecting pieces and friction in the kinematic pair; 5) Model optimization and environment customization: Parameters can be optimized to make the whole model more reasonable. Users can directly conduct dialogues or customize menus. The specific modeling procedures are shown in Fig. 2. 


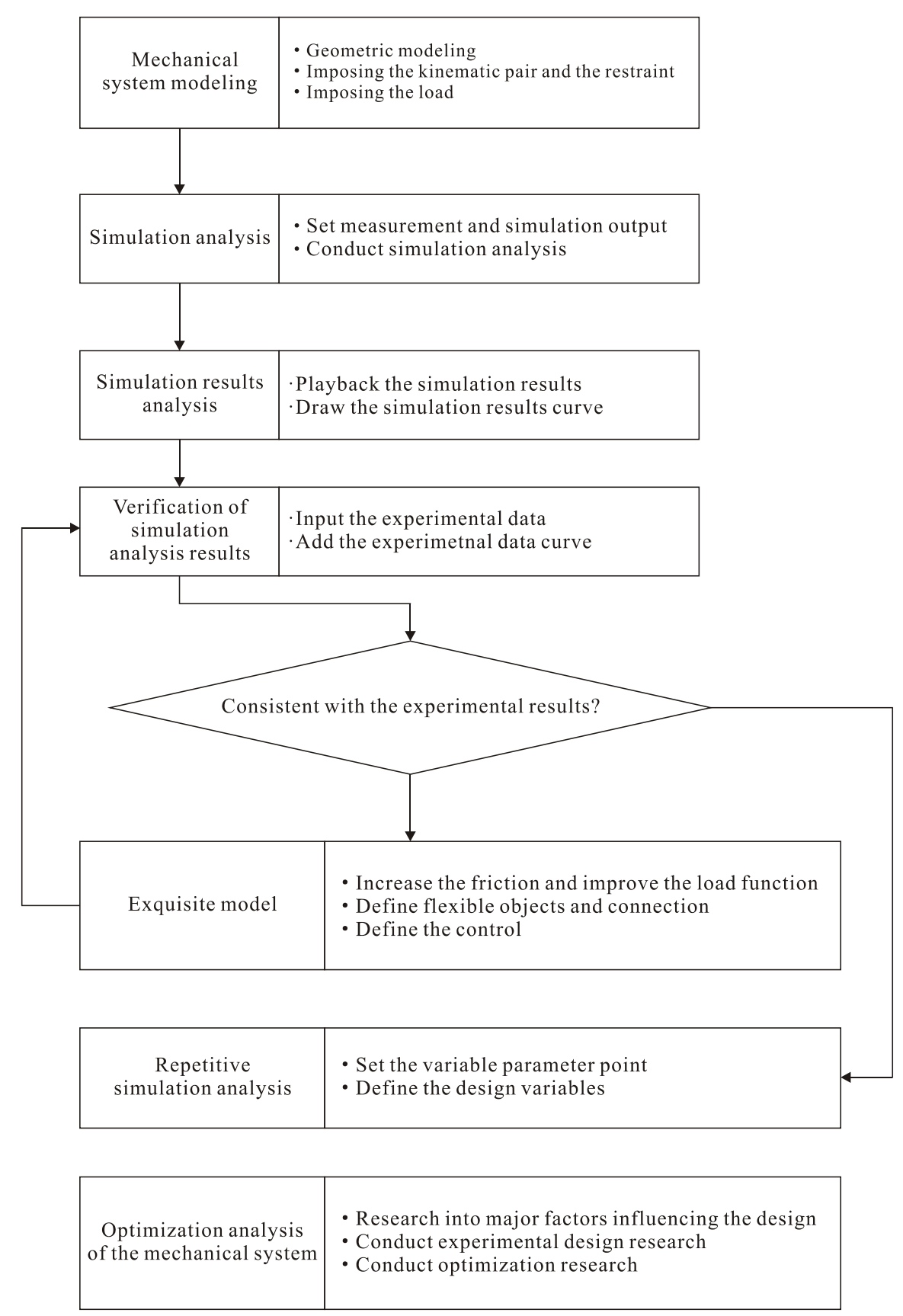

Fig. 2 Simulation flow diagram

Based on the simulation, the kinematic modeling process should be considered. During the operation process, the tool magazine of the ADAMAS should be employed. In this way, the inspection robot model under the environment can be obtained. See Fig. 3 below. 


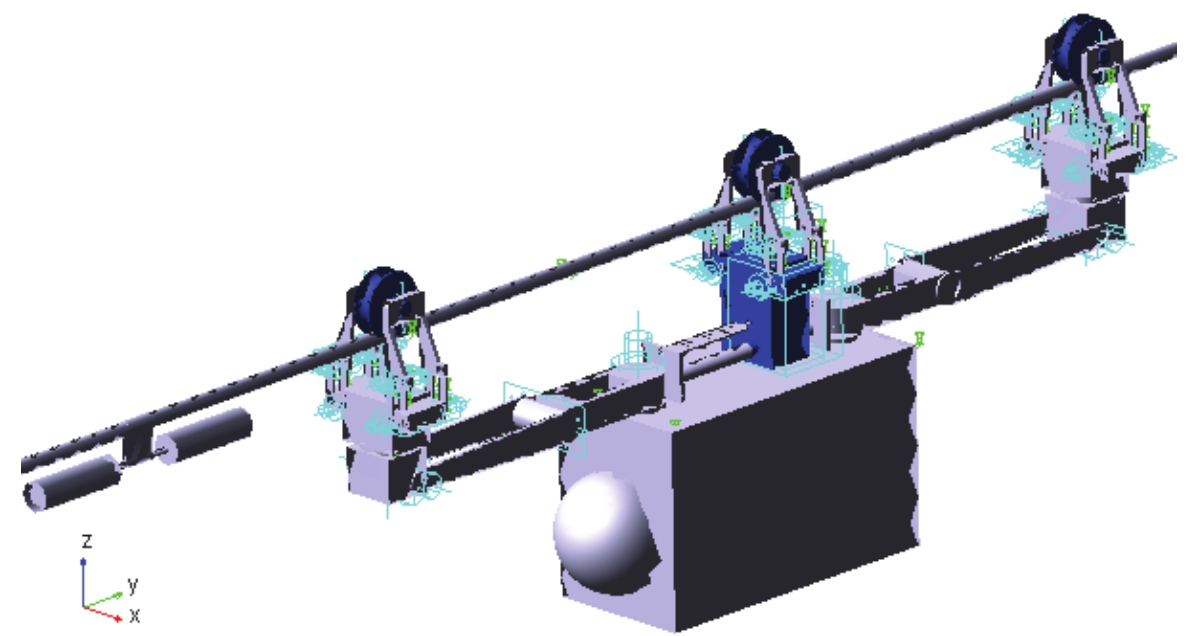

Fig. 3 Robot model under the ADAMAS environment

Through simulation and model building, the specific conditions during the practical joint movements can be obtained. Based on that, the kinematic rules of all robot parts are analyzed. Under the faulted condition, the corresponding rotational angle can be measured. Besides, during the process of evading obstacles, the falling and rising of the mechanical arms can be deduced [4].

\section{Conclusions}

Application of the inspection robot is an important approach to improve the current ultra-high voltage power transmission test level. The structural design of the inspection robot is critical to the application process. The structure should meet the transmission lien requirements. Besides, the robot structure should be simulated so as to judge whether relevant parts meet the practical operation requirements. Only in this way, functions of the inspection robot can be given into full play.

\section{References:}

[1]LI Yong. Research into the application mode of the unmanned aerial vehicle during the inspection of ultra-high voltage AC power transmission line[D]. North China Electric Power University, 2014.

[2]ZHANG $\mathrm{Hu}$. The research and implementation on control system of the flight-sliding type transmission line inspection robot[D]. North China Electric Power University, 2014.

[3]SHI Huiwen. Design and analysis of broken strands reposition metamorphic mechanism of extra-high-voltage power transmission lines[D]. Northeastern University, 2012.

[4]XIANG Xiangfei. System design and simulation study of inspection robot for extra-high voltage power transmission lines[D]. Changchun University of Science and Technology, 2012. 